\section{Modelo de ficha clínica ortodóntica en pacientes con fisura labio palatina}

\section{Application of a novel orthodontic medical record in cleft lip and palate patients}

\section{Resumen}

La ficha clínica es un documento médico legal que permite el registro de la información de un paciente y su problema de salud. En la Unidad de Malformaciones Craneofaciales de la Universidad de Chile, se ha utilizado por 33 años un modelo único de ficha clínica dental utilizado específicamente para la atención ortodóntica en pacientes con fisura labio palatina. El objetivo de este trabajo, es exponer una ficha clínica ortodóntica utilizada con finalidades tanto de documento clínico y docencia.

Palabras clave: Ficha clínica; Ortodoncia; Labio hendido; Fisura del paladar (fuente: DeCS BIREME).

\begin{abstract}
The medical record is a medico-legal document that allows the registration of a patient and his/her health problem. During the last 33 years, a unique model of orthodontic medical record has been used specifically for orthodontic care in patients with cleft palate at the Craniofacial Malformation Unit of the University of Chile. The objective of this work is to present an orthodontic medical record that can be used for both, clinical and teaching purposes.
\end{abstract}

Keywords: Medical record; Orthodontics; Cleft lip; Cleft palate (source: MeSH NLM).

\section{Nota Científica}

\author{
Noemi Leiva ${ }^{1, a, b}$, Vanessa Fuentes ${ }^{1, a}$, Francisca Ayala ${ }^{1, a}$, \\ Carolina Stange ${ }^{1, a}$ \\ ${ }^{1}$ Universidad de Chile, Facultad de Odontología, Unidad \\ de Malformación Cráneofacial, Santiago, Chile. \\ ${ }^{a}$ Cirujana Dentista. \\ ${ }^{\mathrm{b}}$ Especialista en Ortodoncia y Ortopedia Maxilofacial.

\section{Correspondencia:} \\ Vanessa Fuentes \\ Correo electrónico: vane.fuentes.f@gmail.com \\ General Holley 2381 oficina 606, torre B, Providencia, \\ Santiago, Chile.
}

\section{Coautores:}

Noemi Leiva

leivanoemi@yahoo.com

Francisca Ayala

francisca@ayalajimenez.com

Carolina Stange

carostange@gmail.com

\section{Editor invitado:}

Juan Carlos Cuevas-González

Universidad Autónoma de Ciudad Juárez, México.

Conflicto de intereses: los autores declaran no tener conflictos de interés.

\section{Fuente de financiamiento: ninguna.}

\section{Recibido: 25/03/2019}

Aceptado: 04/05/2019

Publicado: 12/09/2019 


\section{Introducción}

La ficha clínica es un documento médico legal, que registra de forma cronológica toda la información sobre un paciente y su problema de salud. Para esto, es necesario recolectar datos personales y familiares, antecedentes de enfermedades y situación actual, que sean relevantes para determinar el diagnóstico más certero y así, un plan de tratamiento efectivo. La ficha clínica no solo sirve como expediente clínico, sino que tiene otros objetivos como la docencia e investigación, gestión y administración, epidemiología e instrumento legal ${ }^{1}$. Su registro es confidencial, su utilidad se reserva al paciente, el establecimiento y sus tratantes, que deben guardar el secreto profesional. Para la revelación de su contenido es necesario el consentimiento o solicitud del paciente; o puede ser el director del establecimiento quien reciba una orden judicial para solicitar la información ${ }^{2}$.

Para la confección de una ficha clínica odontológica, es importante seguir un modelo sistemático, para evitar el riesgo de no obtener la información necesaria ${ }^{3}$.

En la Unidad de Malformaciones Craneofaciales de la Universidad de Chile, desde sus inicios hace 33 años, se ha utilizado un modelo único y propio de ficha clínica enfocado al tratamiento ortodóntico en pacientes con fisura labio palatinas u otras alteraciones del territorio cráneo facial. Este registro clínico ha sido confeccionado por la necesidad de abarcar todos los factores concluyentes para determinar el plan de tratamiento para un paciente con fisura, recolectando información clave como el análisis profundo con la presencia de anomalías del paciente y/o familiares, la exposición a factores químicos-físicos-biológicos durante el embarazo y una clasificación propia para el diagnóstico certero de fisuras labio palatinas. El objetivo de este trabajo es exponer una ficha clínica ortodóntica utilizada con finalidades tanto de documento clínico y docencia.

\section{La ficha clínica}

En la Tabla se presenta el modelo de ficha clínica de la Unidad de Malformaciones Craneofaciales de la Facultad de Odontología de la Universidad de Chile.

En la primera parte de la ficha clínica ortodóntica expuesta, se registran datos de identificación del paciente de manera completa incluyendo datos de origen étnico, lugar donde nació, quienes conforman su grupo familiar, actividades de sus padres y el diagnóstico con el que el paciente ha ingresado. Esta información solicitada tiene el propósito de recolectar la mayor cantidad de factores genéticos y/o ambientales que se pueda informar. Respecto de la etnia, en general las poblaciones amerindias y asiáticas presentan mayor frecuencia en casos de fisuras orofaciales y en menor "grado" las poblaciones caucásicas y africanas ${ }^{4,5}$. En un estudio de prevalencia realizado en el Hospital Félix Bulnes de Santiago 6, se encontró que el mayor porcentaje de pacientes afectados con fisura presentaban etnia amerindia, determinado por examen sanguíneo y la prevalencia del alelo $\mathrm{O}$ y alelo $\mathrm{D}(\mathrm{Rh}+)$. Existen elementos dentro de este registro que exponen la condición socio económica del paciente como el lugar donde nació, edad de los padres, número de hijos, con quien vive el paciente, escolaridad, actividad de los padres y del paciente ${ }^{5,7}$. Estos datos permiten evaluar el nivel biopsicosocial del sujeto, temática relevante para la evaluación de disponibilidad a los servicios de salud durante el embarazo, la asociación a hábitos saludables, exposición a alcohol y consumo de cigarro o drogas 5,7 .

Las edades de los padres es un dato importante que se ha investigado para evaluar su relación con la presencia de fisuras orales. Padres de 40 años o mayores tienen mayor probabilidad de tener hijos con fisuras labio-palatinas. En el caso de las madres entre 35-39 años, la posibilidad de hijos con fisura palatina aumenta y sobre 40 años la probabilidad es mayor de fisuras labiales con o sin inclusión del paladar ${ }^{4,7}$.

Existen diferentes y variadas taxonomías de fisuras orales. En el registro clínico de la Unidad se utiliza la clasificación anatómica distinguiendo así: fisuras de labio, fisuras de paladar, fisura de labio-paladar y fisuras atípicas. Esta organización, nomina la fisura según el sitio morfológico donde se produce ${ }^{8}$, lo que permite la simplificación de su diagnóstico.

La siguiente parte de la ficha corresponde al registro de antecedentes familiares, de otras malformaciones que se puedan presentar en la familia y los parientes.

En cuanto a la consulta sobre otro sujeto del grupo familiar que se encuentre afectado/a con algún tipo de malformaciones, permite evaluar el carácter genético de éste. Se ha encontrado una relación positiva en los casos de fisuras orales dentro de la historia familiar, lo que representa un factor de riesgo para este tipo de malformaciones ${ }^{6,7}$.

El tercer segmento de la ficha corresponde a la inspección de los antecedentes ambientales, debido a su importancia en la etiología de la malformación, son estos factores los que interactúan con genes alterados ${ }^{9}$.

Los factores de riesgo asociados a la presencia de fisuras orales, son diversos, y se han distribuido en el registro clínico separándolos en enfermedades agudas, crónicas, inmunización, factores físicos, químicos y psíquicos. En relación a agentes químicos, se refiere al consumo de fármacos. Es conocido que el consumo de éstos durante el embarazo debe ser restringido y cuidadoso. Algunos de los cuales están asociados a aumentar el riesgo de fisuras orales, tales como los broncodilatadores, hipotensores y anticonvulsivantes ${ }^{6}$.

Respecto de las enfermedades crónicas, existe evidencia que la obesidad, diabetes mellitus y consumo de cigarro durante la gestación, presentan una relación con la probabilidad de desarrollar fisuras orales ${ }^{7,9-11}$. En cuanto a las enfermedades agudas, corresponden a exposiciones a infecciones virales o bacterianas que pueden aparecer durante el período de gestación. Uno de los elementos más relevantes de estas enfermedades son los aumentos de la temperatura que pueden provocar. Se ha encontrado asociación entre estados febriles durante los primeros 
Tabla. Ficha de pacientes portadores de malformaciones congénitas maxilo-faciales

\begin{tabular}{|c|c|c|}
\hline I. & \multicolumn{2}{|l|}{ Identificación } \\
\hline $\begin{array}{l}1 . \\
2 . \\
3 . \\
4 . \\
5 . \\
6 . \\
7 . \\
7 . \\
8 .\end{array}$ & $\begin{array}{l}\text { Número de ficha: } \\
\text { Número de modelos: } \\
\text { Fecha de examen: } \\
\text { Nombre: } \\
\text { Fecha de nacimiento: } \\
\text { Edad: } \\
\text { Sexo: } \\
\text { Dirección: }\end{array}$ & \\
\hline 9. & 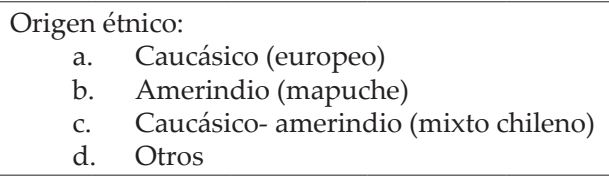 & $\begin{aligned} & \text { 10. Grupo sanguíneo: } \\
& \text { a. } \text { Grupo A } \\
& \text { b. } \text { Grupo B } \\
& \text { c. } \text { Grupo AB } \\
& \text { d. } \text { Grupos 0 } \\
&\end{aligned}$ \\
\hline 11. & $\begin{array}{l}\text { Factor Rh: } \\
\text { a. Rh+ } \\
\text { b. Rh- } \\
\text { c. No sabe }\end{array}$ & 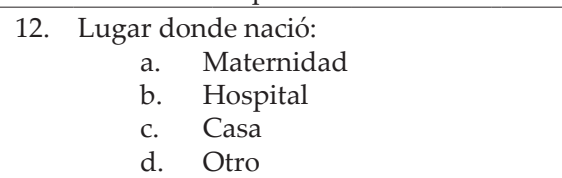 \\
\hline $\begin{array}{l}13 . \\
14 . \\
15 . \\
16 . \\
17 . \\
18 .\end{array}$ & $\begin{array}{l}\text { Región y ciudad donde nació: } \\
\text { Tiempo de gestación al nacer (semanas): } \\
\text { Edad de la madre (nacimiento): } \\
\text { Edad del padre (nacimiento): } \\
\text { Número de hijos (incluyendo al propossitus): } \\
\text { Número de orden en la hermandad: }\end{array}$ & $\begin{array}{l}\text { 19. Con quién vive: } \\
\text { a. Ambos padres } \\
\text { b. Padre } \\
\text { c. Madre } \\
\text { d. Abuelos } \\
\text { e. Hermano/a } \\
\text { f. Otros } \\
\text { 20. Comentarios: }\end{array}$ \\
\hline \multicolumn{3}{|c|}{ 21. Diagnóstico } \\
\hline & A.1 Muesca de labio izquierda & B.1 Muesca de labio derecha \\
\hline & A.2 Labio leporino incompleto izquierdo & B.2 Labio leporino incompleto derecho \\
\hline & A.3 Labio leporino completo izquierdo & B.3 Labio leporino completo derecho \\
\hline & \multicolumn{2}{|l|}{ A.4 Labio leporino y reborde alveolar izquierdo } \\
\hline & \multicolumn{2}{|l|}{ A.5 Labio leporino y reborde alveolar derecho } \\
\hline & \multicolumn{2}{|l|}{ A.6 Labio leporino y reborde alveolar bilateral } \\
\hline & \multicolumn{2}{|c|}{ A.7 Labio leporino y reborde alveolar derecho unilateral complicado con fisura velo palatina } \\
\hline & \multicolumn{2}{|c|}{ A.8 Labio leporino y reborde alveolar izquierdo unilateral complicado con fisura velo palatina } \\
\hline & \multicolumn{2}{|c|}{ A.9 Labio leporino y reborde alveolar bilateral complicado con fisura velo palatina } \\
\hline & \multicolumn{2}{|l|}{ A.10 Fisura de paladar duro } \\
\hline & \multicolumn{2}{|l|}{ A.11 Fisura de paladar blando } \\
\hline & \multicolumn{2}{|l|}{ A.12 Fisura de paladar duro y blando } \\
\hline & \multicolumn{2}{|l|}{ A.13 Fisura submucosa } \\
\hline & \multicolumn{2}{|l|}{ A.14 Úvula bífida } \\
\hline & \multicolumn{2}{|l|}{ A.15 Fisura submucosa y úvula bífida } \\
\hline & \multicolumn{2}{|l|}{ A.16 Atípicas } \\
\hline 22. & 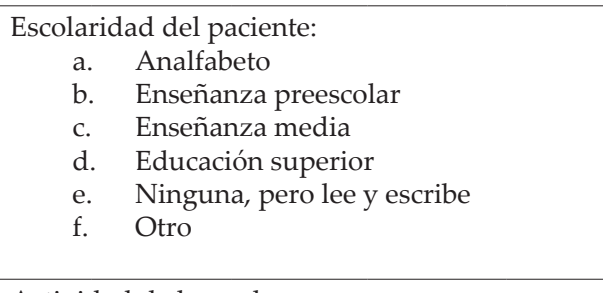 & $\begin{array}{l}\text { 23. Actividad del paciente: } \\
\text { a. } \text { Estudiante } \\
\text { b. Ocupado ocasionalmente } \\
\text { c. Obrero } \\
\text { d. Comerciante } \\
\text { e. Profesional } \\
\text { f. Cesante } \\
\text { g. Otros }\end{array}$ \\
\hline & $\begin{array}{cl}\text { Actividad de la madre: } \\
\text { a. } & \text { Desconocida } \\
\text { b. } & \text { Dueña de casa } \\
\text { c. } & \text { Cesante } \\
\text { d. } & \text { Obrera } \\
\text { e. } & \text { Empleada } \\
\text { f. } & \text { Profesional } \\
\text { g. } & \text { Jubilada } \\
\text { h. } & \text { Fallecida } \\
\text { i. } & \text { No aplicable } \\
\text { j. } & \text { Otros } \\
\end{array}$ & $\begin{array}{ll}\text { 25. Actividad del padre: } \\
\text { a. } & \text { Desconocida } \\
\text { b. } & \text { Cesante } \\
\text { c. } & \text { Obrero } \\
\text { d. } & \text { Empleado } \\
\text { e. } & \text { Profesional } \\
\text { f. } & \text { Jubilado } \\
\text { g. } & \text { Fallecido } \\
\text { h. } & \text { No aplicable } \\
\text { i. } & \text { Otros }\end{array}$ \\
\hline
\end{tabular}


Tabla. Continuación

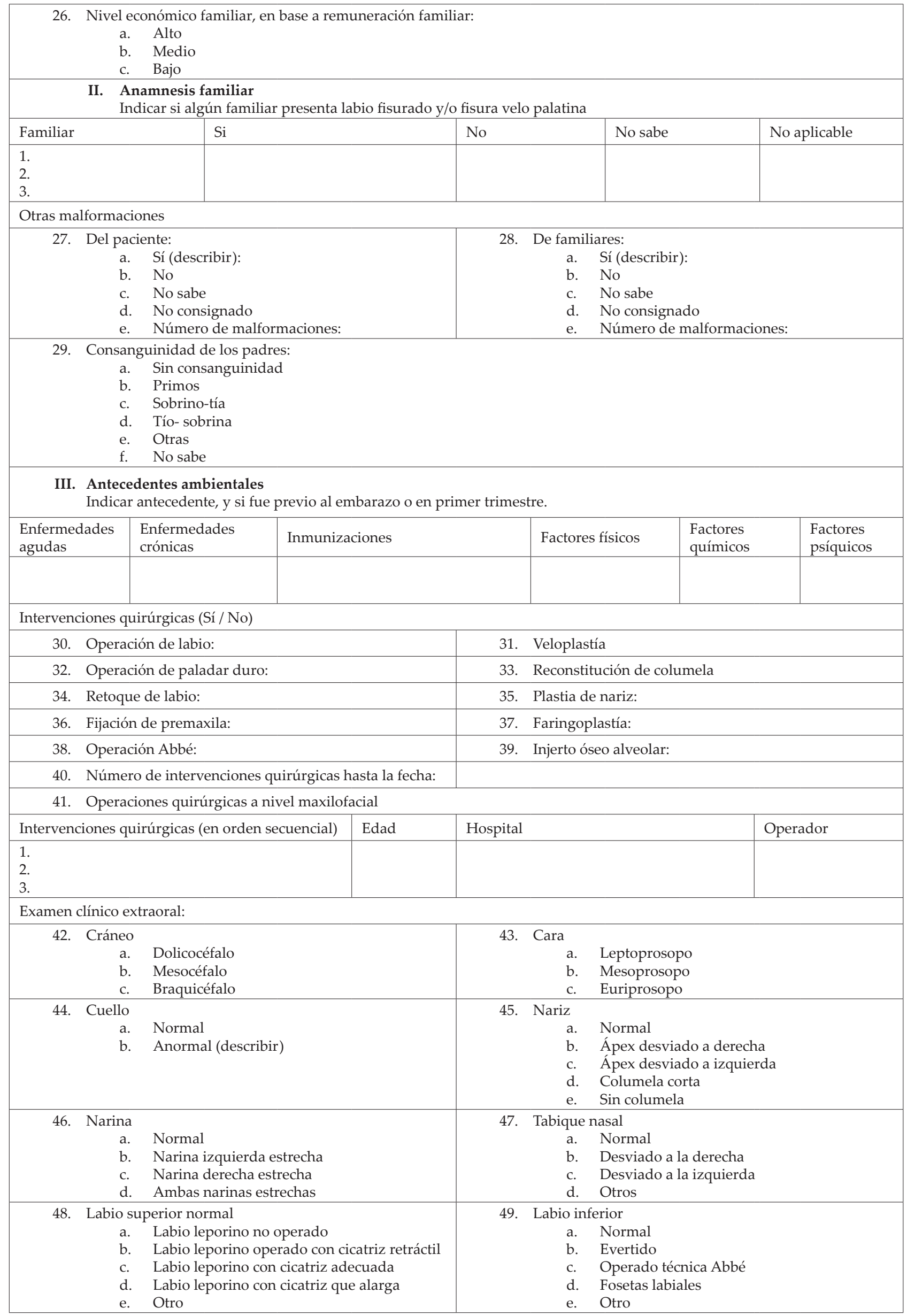


Tabla. Continuación

\begin{tabular}{|c|c|}
\hline $\begin{array}{l}\text { 50. Oclusión labial } \\
\text { a. Ocluye } \\
\text { b. No ocluye } \\
\text { c. Ocluye parcialmente } \\
\text { d. Otro }\end{array}$ & $\begin{aligned} & \text { 51. Examen de ATM } \\
& \text { a. } \text { Normal } \\
& \text { b. } \text { Alterada } \\
& \text { c. } \text { Otro }\end{aligned}$ \\
\hline \multicolumn{2}{|l|}{ IV. Examen clínico intraoral } \\
\hline \multicolumn{2}{|l|}{ Tejidos blandos } \\
\hline 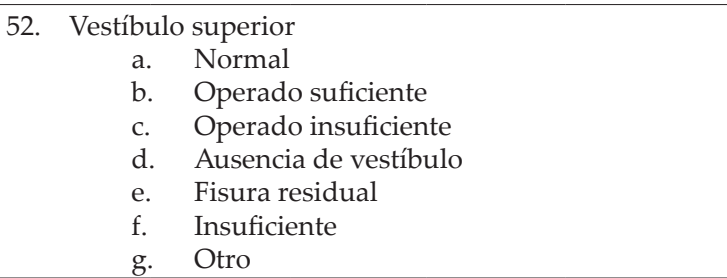 & $\begin{array}{l}\text { 53. Frenillo labial superior } \\
\text { a. Normal } \\
\text { b. Operado adherido a encía suficiente } \\
\text { c. Operado adherido a encía insuficiente } \\
\text { d. Adherido a encía sin operar } \\
\text { e. Otro }\end{array}$ \\
\hline $\begin{array}{l}\text { 54. Espacio en mm entre los rebordes de la fisura } \\
\text { a. Lado izquierdo: } \\
\text { b. Lado derecho: }\end{array}$ & $\begin{aligned} \text { 55. Vestíbulo inferior } \\
\begin{aligned} \text { a. } & \text { Normal } \\
\text { b. } & \text { Anormal }\end{aligned}\end{aligned}$ \\
\hline $\begin{aligned} & 56 . \text { Paladar } \\
& \text { a. } \text { Normal } \\
& \text { b. } \text { Fisurado } \\
& \text { c. } \text { Operado } \\
& \text { d. } \text { Operado con fisura residual } \\
& \text { e. } \text { Operado con perforación residual } \\
& \text { f. } \text { Otro } \\
&\end{aligned}$ & $\begin{array}{ll}\text { 57. Velo } & \\
\text { a. } & \text { Normal } \\
\text { b. } & \text { No operado } \\
\text { c. } & \text { Operado insuficiente } \\
\text { d. } & \text { Operado suficiente } \\
\text { e. } & \text { Otro }\end{array}$ \\
\hline $\begin{array}{ll}\text { 58. Amígdalas palatinas } \\
\text { a. Normal } \\
\text { b. Hipertróficas sanas } \\
\text { c. Hipertróficas infectadas } \\
\text { d. Operadas } \\
\text { e. } & \text { Otro } \\
\end{array}$ & 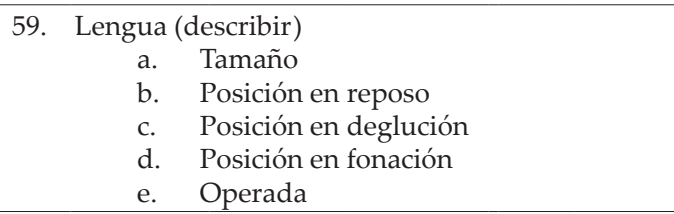 \\
\hline \multicolumn{2}{|l|}{ Tejidos duros } \\
\hline $\begin{array}{l}\text { 60. Dentición: } \\
\begin{aligned} \text { a. } & \text { Lactante } \\
\text { b. } & \text { Temporal } \\
\text { c. } & \text { Mixta primera fase } \\
\text { d. } & \text { Mixta segunda fase } \\
\text { e. } & \text { Permanente } \\
\text { f. } & \text { Desdentada parcial } \\
\text { g. } & \text { Desdentado total }\end{aligned}\end{array}$ & $\begin{array}{ll}\text { 61. Anomalías dentarias: } \\
\text { a. } & \text { Ausentes } \\
\text { b. } & \text { Conoide } \\
\text { c. } & \text { Giroversión } \\
\text { d. Hipoplasias } \\
\text { e. } & \text { Extracción prematura } \\
\text { f. } & \text { Persistencia de temporal } \\
\text { g. } & \text { Extracción permanente } \\
\text { h. } & \text { Estado de resto radicular temporal } \\
\text { i. } & \text { Estado de resto radicular permanente } \\
\text { j. } & \text { Atípico } \\
\text { k. } & \text { Otro: }\end{array}$ \\
\hline \multicolumn{2}{|l|}{$\begin{array}{l}\text { 62. Examen de supernumerarios. } \\
\text { Consignar número de supernumerarios en dentición } \\
\text { temporal y permanente. } \\
\text { - Zona 1: } \\
\text { - Zona 2: } \\
\text { - Zona 3 } \\
\text { - Zona 4: } \\
\end{array}$} \\
\hline \multicolumn{2}{|l|}{ V. Oclusión } \\
\hline $\begin{array}{l}\text { 63. Sentido sagital (nivel canino y molar) } \\
\text { a. } \\
\text { b. Neutroclusión } \\
\text { c. Distoclusión } \\
\text { d. } \\
\text { e. Disto oclusión } \\
\text { Otro }\end{array}$ & $\begin{array}{ll}\text { 64. } & \text { Sentido sagital (nivel incisivo) } \\
\text { a. Normal } \\
\text { b. } & \text { Bis a bis } \\
\text { c. } & \text { Invertida } \\
\text { d. } & \text { Resalte aumentado } \\
\text { e. } & \text { Resalte invertido aumentado } \\
\text { f. } & \text { Otro }\end{array}$ \\
\hline 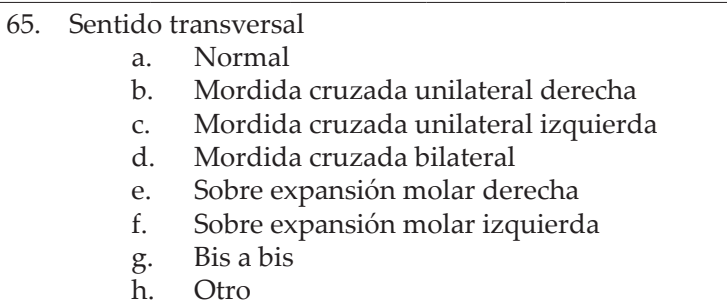 & $\begin{array}{ll}\text { 66. } & \text { Sentido vertical } \\
\text { g. } & \text { Mordida abierta anterior } \\
\text { h. } & \text { Mordida abierta lateral izquierda } \\
\text { i. } & \text { Mordida abierta lateral derecha } \\
\text { j. } & \text { Mordida abierta bilateral } \\
\text { k. } & \text { Bis a bis } \\
\text { l. } & \text { Mordida cubierta } \\
\text { m. } & \text { Sobremordida } \\
\text { n. } & \text { Otro }\end{array}$ \\
\hline
\end{tabular}


Tabla. Continuación

\begin{tabular}{|c|c|c|c|c|}
\hline \multicolumn{3}{|c|}{$\begin{array}{l}\text { 67. En caso de Clase III posición de máxima retrusiva } \\
\text { a. Llega a bis a bis } \\
\text { b. No llega a bis a bis }\end{array}$} & \multicolumn{2}{|c|}{$\begin{array}{l}\text { 68. En caso de Clase II posición de ventaja oclusal } \\
\text { a. Favorable } \\
\text { b. Desfavorable }\end{array}$} \\
\hline \multicolumn{5}{|c|}{$\begin{aligned} \text { 69. Desviación línea media en } \mathrm{mm} \\
\bullet \quad \text { Dentario superior: } \\
\bullet \quad \text { Dentaria inferior: } \\
\bullet \quad \text { Mandibula lado derecho: } \\
\bullet \quad \text { Mandíbula lado izquierdo: }\end{aligned}$} \\
\hline \multicolumn{5}{|c|}{$\begin{array}{l}\text { VI. Exámenes complementarios } \\
\text { En caso de ser realizado indicar fecha. }\end{array}$} \\
\hline \multicolumn{3}{|c|}{ 70. Modelos de estudios iniciales } & \multicolumn{2}{|c|}{ 71. Radiografías retroalveolares } \\
\hline \multicolumn{3}{|c|}{ 72. Radiografía Ortopantomografía } & \multicolumn{2}{|c|}{ 73. Telerradiografía de perfil } \\
\hline \multicolumn{3}{|c|}{ 74. Telerradiografía frontal } & \multicolumn{2}{|c|}{ 75. Cone Beam } \\
\hline \multicolumn{3}{|c|}{ 76. Otras radiografías } & \multicolumn{2}{|c|}{ 77. Fotografías } \\
\hline \multicolumn{5}{|c|}{ Otros tratamientos } \\
\hline \multicolumn{3}{|c|}{ 78. Kinesiológico } & \multicolumn{2}{|c|}{ 79. Fonoaudiológico: } \\
\hline \multicolumn{3}{|c|}{ 80. Psicológico } & \multicolumn{2}{|c|}{ 81. Otro } \\
\hline \multicolumn{5}{|c|}{ VII. Diagnóstico: } \\
\hline \multicolumn{5}{|c|}{$\begin{array}{cl}\text { Diagnóstico de la maloclusión: } \\
\text { a. } & \text { Esqueletal: } \\
\text { b. } & \text { Dentoalveolar: } \\
& \bullet \quad \text { Sagital } \\
& \text { - Transversal } \\
& \text { - Vertical } \\
\text { c. } & \text { Funcional: } \\
\end{array}$} \\
\hline \multicolumn{5}{|c|}{\begin{tabular}{cl}
\multicolumn{2}{l}{ Pronóstico: } \\
$\bullet \quad$ Bueno \\
$\bullet \quad$ Regular \\
• & Malo
\end{tabular}} \\
\hline \multicolumn{5}{|c|}{ Plan de tratamiento } \\
\hline \multicolumn{3}{|l|}{ Etapas } & \multicolumn{2}{|l|}{ Aparatos } \\
\hline \multicolumn{3}{|l|}{$\begin{array}{l}1 . \\
2 . \\
3 .\end{array}$} & & \\
\hline \multicolumn{5}{|c|}{ VIII. Evolución: } \\
\hline Fecha & & Evolución & & Profesional (firma) \\
\hline
\end{tabular}

2 meses de embarazo, que corresponden a la etapa de organogénesis y la aparición de fisuras orales, especialmente cuando no ha existido control alguno sobre la fiebre 7,12. Un elemento que no se ha considerado en la ficha es el consumo de ácido fólico, como factor protector de diversas malformaciones que pueden ocurrir durante la gestación, incluidos los diversos tipos de fisuras orales. Se ha encontrado una disminución en los casos de fisuras orales con el consumo de ácido fólico solo y presente dentro de multivitamínicos previo a la concepción y durante los primeros meses de gestación ${ }^{13,14}$. Es por esto, que su incorporación a la ficha clínica como un factor protector, es de gran importancia.

Una vez finalizada la etapa de la anamnesis, continúa el examen clínico básico extraoral e intraoral, en donde se incluye elementos como evaluación de anomalías dentarias, dientes supernumerarios y examen oclusal, propios de cualquier ficha clínica odontológica.

Si bien para lograr un diagnóstico, el examen clínico es fundamental, los exámenes complementarios tales como radiografías, modelos de estudio e incluso fotografías intraorales, son recursos que permiten registrar el inicio, seguimiento y fin del tratamiento de un paciente. Las fotografías intraorales tienen un uso diagnóstico y médico legal, facilitan el registro de líneas medias, relaciones molares y caninas y permiten evaluar el caso en diferentes períodos de tratamiento hasta su finalización. A diferencia, de los modelos de estudio, capturan, además, información adicional sobre tejidos blandos. Pero su limitación está en el efecto de la angulación durante el registro, que puede afectar las relaciones oclusales en el diagnóstico ${ }^{15}$.

En el caso de los registros imagenológicos, las radiografías son importantes en la determinación de ciertos diagnósticos, para esto el uso de telerradiografías de perfil y el estudio con cefalometrías, son de gran ayuda. Las radiografías retroalveolares y ortopantomografía permiten detectar anomalías dentales, como presencia de supernumerarios, agenesias o incluso dientes que se ubican en la zona de la fisura. Imágenes tridimensionales, como la 
tomografía computarizada, posibilita la evaluación del hueso presente, anomalías dentarias, además se puede usar para el apoyo en el diagnóstico de las alteraciones dentofaciales. Las reconstrucciones tridimensionales logradas permiten la planificación quirúrgica ${ }^{16}$.

En la parte final de la ficha clínica se encuentra el diagnóstico de la maloclusión, describiendo las alteraciones esqueletales, dento alveolares y funcionales. Es necesario realizar un pronóstico y la planificación del tratamiento, que permite de forma ordenada detallar paso a paso cada procedimiento que se debe realizar para la rehabilitación del problema de salud.

\section{Conclusiones}

La fisura labio palatina es una de las malformaciones craneofaciales más comunes. En Chile presenta una prevalencia de 1,4 cada mil nacimientos, según un estudio realizado en 2010 en hospitales chilenos ${ }^{17}$. Su tratamiento no es solo quirúrgico, sino que multidisciplinario, y es necesario considerar la rehabilitación mediante ortodoncia.

La ficha clínica es un elemento necesario para el correcto registro de todos los determinantes que están involucrados en una condición o enfermedad, la información recolectada nos ayuda a formar un diagnóstico preciso y confeccionar un plan de tratamiento.

\section{Referencias bibliográficas}

1. Cuenca K, Rodriguez ML, Soto AD, Pentón O. La historia clínica estomatológica como herramienta en el método clínico y documento médico-legal. Rev Cub Med Mil. 2014;43(4):534-540.

2. Varas J. Ficha clínica: Importancia y trascendencia médico-legal. Rev Obstet. Ginecol. 2011;6(3):235-239.

3. Greenwood M, Essentials of medical history-taking in dental patients. Dent Update. 2015May;42(4):308-315. DOI: $10.12968 /$ denu.2015.42.4.308.

4. Herkrath AP, Herkrath FJ, Rebelo MA, Vettore MV. Parental age as a risk factor for non syndromic oral clefts: A meta analysis. J Dent. 2012Jan;40(1),3-14. DOI: 10.1016/j.jdent.2011.10.002.

5. Kawalec A, Nelke K, Pawlas K, Gerber H. Risk factors involved in orofacial cleft predisposition - review. Open Med. 2015Feb5;10(1):163-175. DOI: $10.1515 / \mathrm{med}-$ 2015-0027.

6. Sepúlveda G, Palomino H, Cortés J. Prevalencia de fisura labiopalatina e indicadores de riesgo: Estudio de la población atendida en el Hospital Clínico Félix Bulnes de Santiago de Chile. Rev Esp Cir Oral y Maxiclofac. 2008;30(1):17-25.

7. Xu DP, Qu WD, Sun C, Cao RY, Liu DW, Du PG. A Study on environmental factors for Nonsyndromic Cleft Lip and/or Palate. J Craniofac Surg. 2018Mar;29(2):364367. DOI: 10.1097/SCS.0000000000004214.

8. Cauvi D, Leiva N, Clasificación de las Fisuras. En: Cauvi D, Leiva $\mathrm{N}$ editores. Etiopatogenia y Tratamiento de las Fisuras Labio- Máxilo- Palatinas. Instituto de Rehabilitación de Malformaciones Máxilo-faciales Universidad de Chile. 2016. p.81-96.

9. Blanco R, Colombo A, Suazo J. Maternal obesity is a risk factor for orofacial clefts: a meta-analysys. $\mathrm{Br} \mathrm{J}$ Oral Maxillofac Surg. 2015Oct;53(8):699-704. DOI: 10.1016/j.bjoms.2015.05.017.

10. Barrera C, Mezarobba N. Maternal Risk Factors Associated with Cleft Lip with or without Cleft Palate: A Review. Int. J. Odontostomat. 2016;10(2):359-368.

11. Xuan Z, Zhongpeng Y, Yanjun G, Jiaqi D, Yuchi Z, Bing S, Chenghao L. Maternal active smoking and risk of oral clefts: a meta-analysis. Oral Surg Oral Med Oral Pathol Radiol. 2016;122(6):680-690. DOI: 10.1016/j. oooo.2016.08.007.

12. Shahrukh Hashmi S, Gallaway MS, Waller DK, Langlois $\mathrm{PH}$, Hecht JT. Maternal fever during early pregnancy and the risk of oral clefts. Birth Defects Research. 2010Mar;88(3):186-194. DOI: 10.1002/bdra.20646.

13. Jahanbin A, Shadkam E, Miri HH, Shirazi As, Abtahi M. Maternal Folic Acid Supplementation and the Risk of Oral Clefts in Offspring. J Craniofac Surg. 2018Sep; 29(6):534-541. DOI: 10. 1097/ SCS.0000000000004488.

14. Millacura N, Pardo R, Cifuentes L, Suazo J. Effects of folic acid fortification on orofacial clefts prevalence: a meta-analysis. Public Health Nutr. 2017Aug;20(12):22602268. DOI: $10.1017 / S 1368980017000878$.

15. Jackson TH, Kirk CJ, Phillips C, Koroluk LD. Diagnostic accuracy of intraoral photographic orthodontic. J Esthet Restor Dent. 2019.Jan;31(1):64-71. DOI: 10.1111/ jerd. 12426.

16. Abramson ZR, Peacock ZS, Cohen HL, Choudhri AF. Radiology of Cleft Lip and Palate: Imaging for the Prenatal Period and throughout Life. Radiographics. 2015. Nov-Dec;35(7):2053-2063. DOI: 10.1148/ rg.2015150050.

17. Nazer J, Cifuentes L. Prevalencia al nacimiento de malformaciones congénitas en las maternidades chilenas participantes en el ECLAMC en el período 2001-2010. Rev Med Chile. 2014;142(9):1150-1156. DOI: $10.4067 /$ s0034-98872014000900009 
